\title{
Unified loss theory and its application on low voltage networks
}

Dr. András Dán (e-mail: dan.andras@,vet.bme.hu), David Raisz

Power Systems and Environment Group, Department of Electric Power Engineering Budapest University of Technology and Economics

\begin{abstract}
This paper presents a unified loss theory, a measurement and computation method for the determination of network losses. This approach enables to jointly treat and compare the different components of losses such as reactive current, time-variance, asymmetry and harmonic currents, thus making it possible to evaluate the effect of different loss-reduction investments. Further a stochastic loss computation method is introduced, and compared to the previously shown method. Finally, measurement results and their evaluation are presented.
\end{abstract}

Keywords

Network losses, harmonics, asymmetry, load factor, Monte-Carlo method, stochastic load models

\section{Introduction}

Considering the present Hungarian network conditions the network losses run up to $10-12 \%$ of the total electric power fed in, and ca. $40 \%$ of these losses arise on the LV networks.

Therefore it is an important task to survey the components of the LV network losses, on the one hand in order to accurately estimate the losses (no widely-used automatic meter reading infrastructure is installed yet), and on the other hand to enable a cost-benefit analysis of loss reduction investments.

The aims of the research reported on are:

- to determine the amount of information needed to estimate the LV network losses in a way as simple and as accurate as possible, and

- to establish a simple measurement procedure and a computation algorithm.

In Section 2 the components of the LV network losses are reviewed

In Section 3 the fundamentals of a unified loss theory are presented, which is further improved in Section 4.

Section 5 describes a Monte-Carlo approach for the network loss calculation.
The load models used in this approach are outlined in the Appendix.

Section 6 shows measurement and simulation results using both the unified theoretical and the Monte-Carlo approach.

\section{Components of $\mathbf{L V}$ network losses}

The unified loss theory proposed in Section 3 comprises the determination of an ideal case network loss arising due to the total energy supplied to the consumers, and the calculation of additional lossincreasing components, such as

- capacity load factor

- fundamental frequency reactive power

- fundamental frequency current asymmetry (negative and zero sequence)

- harmonic currents.

\section{Capacity load factor}

The current $i(t)$ flowing through a line segment with a resistance $R$ from time 0 to $T$ causes a loss of energy

$$
W_{v}=R \cdot \int_{0}^{T} i^{2}(t) \cdot d t \quad[\mathrm{~W} \cdot \mathrm{s}]
$$

In ideal case the current is constant over the time and the loss is expressed:

$$
W_{v(i d)}=T \cdot R \cdot i_{k}^{2} \quad[\mathrm{~W} \cdot \mathrm{s}]
$$

where $i_{k}=\frac{1}{T} \int_{0}^{T} i(t) \cdot \mathrm{dt} \quad[\mathrm{A}]$. The capacity load factor $\mathrm{K}_{\mathrm{cs}}=\left(\frac{W_{v}}{W_{v(i d)}}\right)$ shows the loss increase relative to the ideal case, due to the time-varying current.

\section{Fundamental frequency reactive power}

In the ideal case the consumer current has only an active component $I_{P}$ causing a network loss power of $P_{v(i d)}=3 \cdot R \cdot I_{P}^{2} \quad[\mathrm{~W}]$. If the current $I_{S}$ 
consists of reactive component as well, then the loss power $P_{v}=3 \cdot R \cdot\left|\mathbf{I}_{\mathbf{S}}\right|^{2}[\mathrm{~W}]$, and the loss increase is expressed in eq.3.

$$
\left(\frac{P_{v}}{P_{v(i d)}}-1\right)=\frac{1}{(\cos (\varphi))^{2}}-1
$$

\section{Harmonic currents}

The loss increase due to harmonic currents can be expressed as $\mathrm{P}_{\mathrm{vh}} / \mathrm{P}_{\mathrm{v}(\mathrm{id})}=1+\left(\mathrm{THD}_{\mathrm{I}}\right)^{2}$ for harmonic orders $h$ not being multiples of 3. In case of triplen harmonic currents the additional loss of neutral wire, should be considered as well: $\mathrm{P}_{\mathrm{vn}, \mathrm{h}, 3 .} / \mathrm{P}_{\mathrm{v} 3(\mathrm{id})}=3\left(\mathrm{THD}_{\mathrm{I}}\right)^{2}$.

\section{Asymmetry}

Negative sequence current causes an additional loss only in the phase wires, whereas the zero sequence current produces an additional loss in the phase wires as well as in the neutral wire. Latter is proportional to $9 \mathrm{I}_{0}^{2}$, because the current in the neutral wire is three times the zero sequence current $\mathrm{I}_{0}$.

\section{Aggregate treatment of network loss components: the unified loss theory}

Let us consider a line segment with resistance $\mathrm{R}$, and the current be timevariant, asymmetric and distorted with harmonics.

The loss on this line segment can be written as $\mathrm{P}_{\mathrm{v}}=\mathrm{P}_{\mathrm{a}}+\mathrm{P}_{\mathrm{b}}+\mathrm{P}_{\mathrm{c}}=3 \mathrm{P}_{+}+3 \mathrm{P}_{-}+3 \mathrm{P}_{0}$

where

$\mathrm{P}_{\mathrm{a}}=\left(\mathrm{I}_{\mathrm{a}, \mathrm{rms}}\right)^{2} \mathrm{R}$, etc.

$\mathrm{P}_{+}=\left(\mathrm{I}_{+}\right)^{2} \mathrm{R}_{+}$summarized for positive sequence fundamental and harmonic components

$\mathrm{P}_{-}=\left(\mathrm{I}_{-}\right)^{2} \mathrm{R}$. summarized for negative sequence fundamental and harmonic components

$\mathrm{P}_{0}=\left(\mathrm{I}_{0}\right)^{2} \mathrm{R}_{0}$ summarized for zero sequence fundamental and harmonic components incl. the loss on the earth wire

Denoting the fundamental frequency positive sequence current by $\mathrm{I}_{+}$, the corresponding power factor (computed from $\mathrm{I}_{+}$and the $\mathrm{V}_{+}$) by $\cos \varphi$, then the 3 phase ideal-case loss is $\mathrm{P}_{\mathrm{v}, \mathrm{id}}=3\left(\mathrm{I}_{+} \cos \varphi\right)^{2} \mathrm{R}_{+}$. Let's apply the definition of capacity load factor for the fundamental frequency positive sequence current:

$$
K_{c s}^{+}=\frac{\frac{1}{T} \int_{0}^{T}\left[i^{+}(t)\right]^{2} d t}{\left(\frac{1}{T} \int_{0}^{T} i^{+}(t) d t\right)^{2}}
$$

and similarly for the other sequences and harmonics.

If we assume, that

- $\mathrm{R}+=\mathrm{R}-=\mathrm{R} 0$ (and is the same for all harmonics)

- $\cos \varphi=$ constant over time

- $\quad A_{I-}=\frac{I_{-}}{I_{+}}, \quad A_{I 0}=\frac{I_{0}}{I_{+}} \quad$ asymmetry factors are constant over time

- $\quad D_{I h, s}=\frac{I_{h, s}}{I_{+}} \quad$ harmonic distortion

factors are constant over time, then the ratio of the real-case loss energy to the ideal-case loss energy will be:

$$
\begin{aligned}
& \frac{W_{v}}{W_{i d}}= \frac{K_{c s}^{+}}{\cos ^{2} \varphi} \cdot \\
& \cdot\left(1+\frac{K_{c s}^{-}}{K_{c s}^{+}} A_{I-}^{2}+\frac{K_{c s}^{0}}{K_{c s}^{+}} 4 A_{I 0}^{2}\right. \\
&\left.+\frac{K_{c s}^{I 3,0}}{K_{c s}^{+}} 4 D_{I 3,0}^{2}+\frac{K_{c s}^{I 5,-}}{K_{c s}^{+}} D_{I 5,-}^{2}\right)
\end{aligned}
$$

Using this formula it is possible to estimate the loss of a LV network based on measurements performed solely at the feeding point of the network, if we assume that the measured current is divided among the consumers in the same proportion every moment.

With the above assumptions the formula enables to compare the loss components and to evaluate the effect of different lossreduction investments.

\section{Enhancements of the unified loss theory}


In this section we present how Eq. (5) has to be modified if we assume more realistic conditions. At the same time some factors similar to the above mentioned will be derived that are also easily calculated from measurement data; using these factors it is possible to compare different LV networks and queue loss reduction investments.

Let us consider a LV feeder with $\mathrm{N}$ consumers and $\mathrm{N}$ line segments (see Fig. 1 ), and let us assume that the time-varying complex current $I(t)$ - either harmonic and sequence - measured at the busbar is divided among the consumers so that the current of the consumer $k$ is

$$
I_{k}(t)=\alpha_{k} I(t)
$$

where $\sum_{k=1}^{N} \alpha_{k}(t)=1$ and all $\alpha_{k}$ are constant over time.

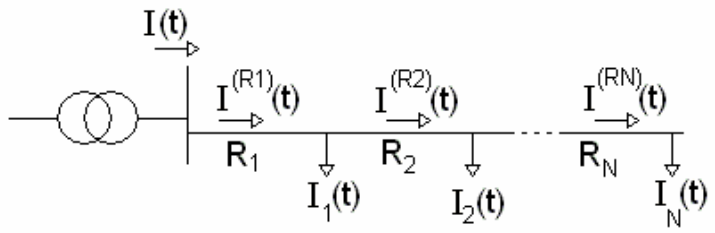

Fig. 1 Sample network

Thus the loss power can be expressed as

$$
\begin{aligned}
& P_{L}(t)=R_{1}\left|I^{R 1}(t)\right|^{2}+R_{2}\left|I^{R 2}(t)\right|^{2}+\ldots+R_{N}\left|I^{R N}(t)\right|^{2}= \\
& =R_{1}\left|\sum_{k=1}^{N} I_{k}(t)\right|^{2}+R_{2}\left|\sum_{k=2}^{N} I_{k}(t)\right|^{2}+\ldots+R_{N}\left|I_{N}(t)\right|^{2}= \\
& =R_{1}|I(t)|^{2}\left(\sum_{k=1}^{N} \alpha_{k}(t)\right)^{2}+R_{2}|I(t)|^{2}\left(\sum_{k=2}^{N} \alpha_{k}(t)\right)^{2}+ \\
& \ldots+R_{N}|I(t)|^{2} \alpha^{2}{ }_{N}(t)= \\
& =|I(t)|^{2} \cdot \bar{R}
\end{aligned}
$$

where

$$
\bar{R}=\sum_{n=1}^{N} R_{n}\left(\sum_{k=n}^{N} \alpha_{k}(t)\right)^{2}
$$

will be called "equivalent resistance" of the LV feeder.

Such equivalent resistances can be calculated for every sequence and harmonic order considered (and it can be calculated for active and reactive parts of the positive sequence fundamental current).

The scale parameters $\alpha_{k}$ for the fundamental frequency positive sequence active current can be chosen according to the annual energy consumption, i.e. $\alpha_{k}=\frac{W_{k}}{\sum_{i=1}^{N} W_{i}}$, where $W_{i}$ is the annual energy consumption of the $i$-th consumer.

For all other current components either the above scale parameters can be used, or - as a first step towards a probabilistic model they can be varied randomly, as long as their sum remains unity. The random variation can be performed based on statistical evaluation of previously recorded measurement data of different typical consumer types. (See Fig. 2 and Fig. 3.)

After determining the parameters $\alpha_{k}$ and the equivalent resistances (8) the loss components can be calculated according to Eq. (7) for every sequence and harmonic order, and the total loss can be expressed as

$$
P_{\text {loss }}=3 \cdot \sum_{h} \sum_{s=0,1,2}\left|I_{s}^{(h)}(t)\right|^{2} \cdot \overline{R_{s}^{(h)}}
$$

By integrating Eq. (9) over time one obtains the loss energy for that time interval, by averaging Eq. (9) over time one obtains the mean loss power.

In order to express the mean loss power as the product of the ideal case loss and the sum of the loss increasing factors, Eq. (9) has to be rearranged. Let us denote $\langle x\rangle=\frac{1}{T} \int_{0}^{T} x(t) d t$ the average of $x(t)$ over time.

Then the ideal loss can be expressed as

$$
P_{i d}=3 \cdot\left\langle I_{1, W}^{(1)}\right\rangle^{2} \overline{R_{1, W}^{(1)}}
$$

and the total mean loss power as 


$$
\begin{aligned}
& P_{\text {loss }}=3 \cdot\left\langle I_{1, W}^{(1)}\right\rangle^{2} \overline{R_{1, W}^{(1)}} .
\end{aligned}
$$

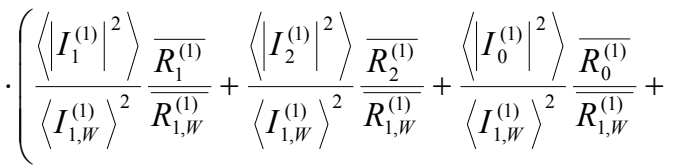

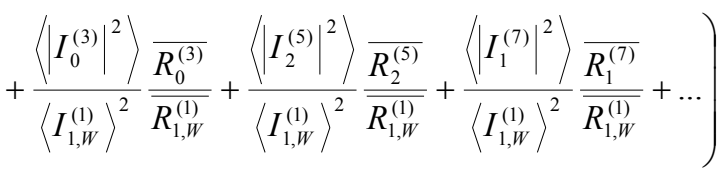

(Note, that in Eq. (11) only the dominant sequence components of the harmonic currents were taken into consideration, i.e. zero sequence for the $3^{\text {rd }}$, negative sequence for the $5^{\text {th }}$ and positive sequence for the $7^{\text {th }}$ harmonic currents, all other components can usually be neglected.)

The quotient in Eq. (11)

$$
\frac{\left\langle\left. I_{1}^{(1)}\right|^{2}\right\rangle}{\left\langle I_{1, W}^{(1)}\right\rangle^{2}}=\frac{\frac{1}{T} \int_{0}^{T} \mid I_{1}^{(1)}(t)^{2} d t}{\left[\frac{1}{T} \int_{0}^{T} I_{1, W}^{(1)}(t) d t\right]^{2}} \quad \text { can be }
$$

considered as the generalization of the loss-increasing effect of both the capacity load factor and $\cos \varphi$ in Eq. (5), since it shows the loss increase due to the presence of an imaginary component and timevarying behavior of the positive sequence fundamental current.

Similarly, $\frac{\left\langle\left. I_{2}^{(1)}\right|^{2}\right\rangle}{\left\langle I_{1, W}^{(1)}\right\rangle^{2}}$ and $\frac{\left\langle\left. I_{0}^{(1)}\right|^{2}\right\rangle}{\left\langle I_{1, W}^{(1)}\right\rangle^{2}}$ can be seen as generalizations of the asymmetry factors, and ratios $\frac{\left\langle\left. I_{s}^{(h)}\right|^{2}\right\rangle}{\left\langle I_{1, W}^{(1)}\right\rangle^{2}}$ for s $=\{0,1,2\}$ and $\mathrm{h}>1$ can be treated as generalizations of the loss-increasing harmonic distortion factors.

The method assumes that the consumers on the network considered are very similar in nature (see Eq. (6)), e.g. only household consumers exist on the network. Nevertheless, this approach can be extended so that it can take into consideration one or more consumers,
- that have probably significantly larger consumption

- the currents of which are a-priori known (the currents can be either measured quasi on-line using AMR or estimated using pre-defined user profiles).

(This extension will not be further detailed in this paper.)

Using Eq. (11) it is possible to calculate the network loss in a simple way using measurement data and some basic information (annual consumption) about customers.

This method is fast, easy to implement, and yields insight into the composition of the loss.

There are, however, some issues that the above method cannot cope with.

Presently in the Hungarian LV distribution system a considerable part of the residential consumers has a single-phase PCC, but the operators' databases lack the information about the exact connection phase of each consumer. This uncertainty cannot be dealt with the method described above in an easy, straightforward way.

The other drawback of the method is that it assumes time-invariant $\alpha_{k}$ parameters, but in reality the measured current at the transformer is the sum of consumer currents that are varying rather dissimilarly every time step (compare Fig. 6 and Fig. 7).

These considerations led to the elaboration of a pseudo-stochastic model, which is described in the next section.

\section{The Monte-Carlo approach}

The load model described in the Appendix is used in a Monte-Carlo simulation, where the network losses are calculated

- assigning different connection phases for single-phase consumers, and

- assuming different time-varying behavior for the consumer currents in every simulation run. 
The random variation of the parameters is performed in such a way that

- the sum of the consumer currents be equal to the measured currents every time step

- basic properties of consumers known in advance (annual energy consumption, distribution of possible $\cos \varphi$ values and harmonic distortion factors) be complied to as much as possible.

Current measurements of several residential customers were carried out in order to determine usual $\cos \varphi$ values and harmonic distortion factors. Empirical cumulative distribution functions of these quantities resulting from the measured households are shown in Fig. 2 and Fig. 3.

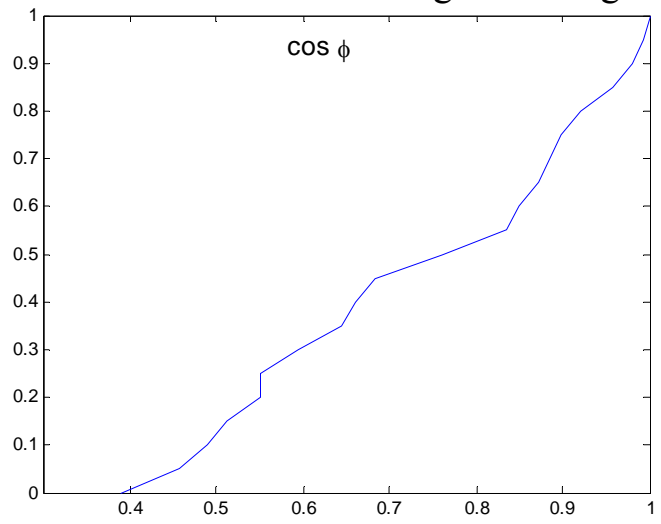

Fig. 2 Empirical cumulative distribution function of $\cos \varphi$ for the measured households

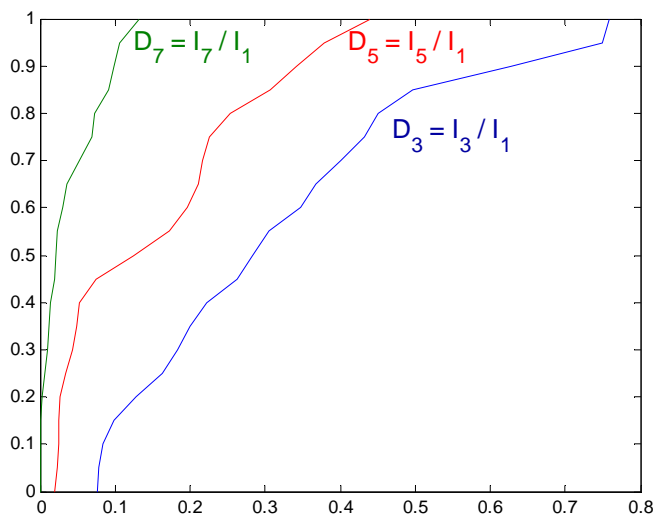

Fig. 3 Empirical cumulative distribution functions of the ratios Dh (mean rms harmonic current to mean rms fundamental current ratio)

In a Monte-Carlo simulation these factors are randomly chosen for every consumer using these distribution functions.
The steps of the Monte-Carlo simulation are outlined below:

1. The connection phase of single-phase consumers is randomly chosen, so that in every phase the sum of the annual consumptions approximately matches the annual energy extrapolated from the phase current measurement data.

2. The consumer currents are composed of so-called "elementary appliances". Thus quite realistic consumer currents can be obtained, but there is no guarantee that the sum of the consumer currents equals the measured currents.

3. Therefore the consumer currents are adjusted using a "Dirichlet distribution" method, so that

a. the sum of the consumer currents equals the measured currents

b. the mean consumer currents comply with the annual energy consumption of the consumer and usual $\cos \varphi$ and harmonic current ratio values.

4. Since the network topology and the resistance values of the line segments are known, the losses can now be calculated.

5. Steps 1-4. are repeated several times, so that the distribution of the network loss results can be statistically evaluated and reported.

\section{Measurement and calculation results}

Two characteristic results of network measurements are reported on in this section.

\section{Network 1}

The topology of Network 1 is presented in Fig. 5 (Line lengths are given in meters.)

Consumers 1, 3, 14 and 20 are larger consumers, which make up for $65 \%$ of the total annual consumption of the consumers. (Post office, shop, bank office, public library.) All other loads are household consumers.

The evaluation of the measurements at the transformer yields that the average positive sequence fundamental frequency active current is 
$\left\langle I_{1, W}^{(1)}\right\rangle=33.6 \mathrm{~A}$, and the factors in Eq. (11)

$$
\frac{\left\langle I_{s}^{(h)}{ }^{2}\right\rangle}{\left\langle I_{1, W}^{(1)}\right\rangle^{2}} \text { are as follows: }
$$

TABLE I. Loss-increasing factors for different sequences $(s)$ and harmonics $(h)$

\begin{tabular}{|c|c|c|c|c|}
\hline $\begin{array}{c}\mathrm{h} \rightarrow \\
\mathrm{s} \downarrow\end{array}$ & 1 & 3 & 5 & 7 \\
\hline 0 & 0.0359 & 0.00573 & 0.000148 & $5.70 \mathrm{e}-005$ \\
\hline 1 & 1.3965 & 0.00043 & 0.000261 & 0.000547 \\
\hline 2 & 0.0262 & 0.00031 & 0.001102 & $2.74 \mathrm{e}-005$ \\
\hline
\end{tabular}

The results obtained by the method according to Eq. (11), (without the extension for large uncommon consumers with a-priori known currents) are as follows:

the ideal case loss calculates to $261 \mathrm{~W}$, the real case loss to $384 \mathrm{~W}$.

More accurate results can be expected from using the Monte-Carlo method. TABLE II. summarizes the results:

TABLE II. Results of Monte-Carlo simulations

\begin{tabular}{|c|r|r|r|r|r|}
\hline W & TLL & CCOT & FCOA & FONH & NL \\
\hline Max: & 477 & 408 & 328 & 467 & 108 \\
P95: & 472 & 405 & 328 & 463 & 101 \\
Mean: & $\mathbf{4 5 8}$ & $\mathbf{3 9 3}$ & $\mathbf{3 2 3}$ & $\mathbf{4 4 8}$ & $\mathbf{8 4}$ \\
Std.dev.: & $\mathbf{1 0}$ & $\mathbf{8}$ & $\mathbf{3}$ & $\mathbf{1 0}$ & $\mathbf{1 2}$ \\
P05: & 443 & 384 & 318 & 435 & 70 \\
Min: & 442 & 384 & 316 & 434 & 66 \\
\hline MTPC & \multicolumn{5}{|c|}{$23 \mathbf{5 7 1}$} \\
\hline
\end{tabular}

where

TTL: Total Line Loss

CCOT: all Currents Constant Over Time

FCOA: Fundamental Current has Only an Active component

FONH: Fundamental Only, No Harmonics NL: $\quad$ loss on the Neutral Line

MTPC: Mean Total Power Consumed

It can be observed, that the two methods give significantly different results.

Network 2

Network 2 consists of 77 household consumers, 22 line segments with a total length of $762 \mathrm{~m}$.

The evaluation of the measurements at the transformer yields that the average positive sequence fundamental frequency active current is

$\left\langle I_{1, W}^{(1)}\right\rangle=52.4 \mathrm{~A}$, and the factors

$\frac{\left.\left|I_{s}^{(h)}\right|^{2}\right\rangle}{\left(I_{1, W}^{(1)}\right\rangle^{2}}$ in Eq. (11) are as follows:

TABLE III. Loss-increasing factors for different sequences $(s)$ and harmonics( $h)$

\begin{tabular}{|c|c|c|c|c|}
\hline $\begin{array}{c}\mathrm{s} \downarrow \\
\mathrm{h} \rightarrow\end{array}$ & 1 & 3 & 5 & 7 \\
\hline 0 & $\mathbf{0 . 0 9 7 4}$ & $\mathbf{0 . 0 1 4 2 0}$ & 0.000403 & $5.49 \mathrm{e}-005$ \\
\hline 1 & $\mathbf{1 . 1 8 0 9}$ & 0.00160 & 0.000535 & $\mathbf{0 . 0 0 0 6 0 7}$ \\
\hline 2 & $\mathbf{0 . 1 1 0 1}$ & 0.00227 & $\mathbf{0 . 0 0 3 0 6 0}$ & 0.000104 \\
\hline
\end{tabular}

The results obtained by the method according to Eq. (11), (without the extension for large consumers with a-priori known currents) are as follows:

the ideal case loss calculates to $1493 \mathrm{~W}$, the real case loss to $2121 \mathrm{~W}$.

The results of the Monte-Carlo method are summarized in TABLE IV:

TABLE IV. Results of Monte-Carlo simulations

\begin{tabular}{|c|r|r|r|r|r|}
\hline W & TLL & CCOT & FCOA & FONH & NL \\
\hline Max: & 2400 & 2168 & 2121 & 2354 & 810 \\
P95: & 2365 & 2126 & 2097 & 2314 & 771 \\
Mean: & $\mathbf{2} 221$ & $\mathbf{1} 967$ & $\mathbf{1} 981$ & $\mathbf{2} 169$ & $\mathbf{6 0 0}$ \\
Std.dev.: & $\mathbf{7 9}$ & $\mathbf{8 8}$ & $\mathbf{6 9}$ & $\mathbf{8 0}$ & $\mathbf{9 6}$ \\
P05: & 2148 & 1895 & 1902 & 2097 & 505 \\
Min: & 2144 & 1894 & 1893 & 2092 & 491 \\
\hline MTPC & \multicolumn{5}{|c|}{$36 \mathbf{6 3 1}$} \\
\hline
\end{tabular}

It can be observed, that in case of Network 2 the two methods give almost identical results, since no large uncommon consumers are present.

\section{Conclusions}

In this paper a unified loss theory was presented as well as a direct and a stochastic simulation and computation procedure for the evaluation of network losses, based on the theory and measured data. Comparison of results of direct and stochastic methods (based on real-life measurement data) reveals that a simple form of the method yields very good results when the consumers all exhibit similar behavior. 
The result of the research reported on is a measurement methodology and a loss evaluation software using input data as follows:

- network topology with the distribution line parameters

- annual energy consumption of consumers $(\mathrm{kWh})$

- consumer connection information (single phase or three-phase)

- current of large consumers (compared to the average consumer, e.g. shops, restaurants, etc.) measured or estimated based on long term profiles

- currents at the feeding point of the LV network measured for at least 1 week with a sampling time of 1 minute (fundamental and harmonic currents, every sequence)

The outputs of the software are the probability density function of the different loss components and the total loss on the feeder.

\section{Acknowledgment}

The authors wish to express their thanks for the financial support of the research to the Hungarian utility companies: E.on DÉDÁSZ Zrt, ELMÜ Nyrt, DÉMÁSZ Nyrt.

\section{References}

[1] A.Dán, D.Raisz, L.Hornyák, S.Rios: Power considerations and digital energy metering accuracy on distorted network, 48. Internationales Wissenschaftliches Kolloquium, TU Ilmenau, 22.-25. September 2003

[2] Dougal H. O. McQueen, Patrick R. Hyland, and Simon J. Watson: Monte Carlo Simulation of Residential Electricity Demand for Forecasting Maximum Demand on Distribution Networks,IEEE TR. on Power Systems, Vol. 19, No. 3, August 2004

[3] A. Capasso, W. Grattieri, R. Lamedica, A. Prudenzi: A Bottom-up Approach to Residential Load Modeling, IEEE Tr. on Power Systems, Vol. 9, No. 2, May 1994

[4] http://en.wikipedia.org/wiki/Dirichlet_distribution

\section{Appendix}

\section{Usage of "elementary appliances"}

Current measurements of several residential customers were carried out in order to reveal the stochastic nature of such load. During the evaluation of these measurements so-called "elementary appliances" were identified, which are different load patterns that the measured household currents can be composed of. Some of these "elementary appliances" are in fact household devices (e.g. the load pattern of a fridge could be identified in most households), others merely represent some electricity usage behavior.

Several - more or less automated - data processing methods have been worked out to identify the "elementary appliances" from measurements, which are not further detailed in this paper.

Once the "elementary appliances" are identified, their statistical properties can be saved in form of empirical cumulative distribution functions for later use. The statistical properties used are as follows:

- distribution function of number of events a day

- distribution of switch-on instants during a day

- distribution function of duration of usage

- magnitude and phase distribution for all harmonics considered. 


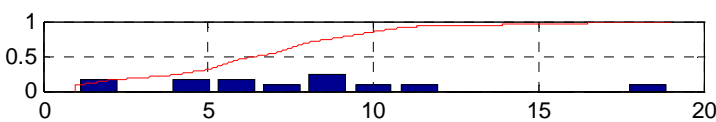

No. of events per day

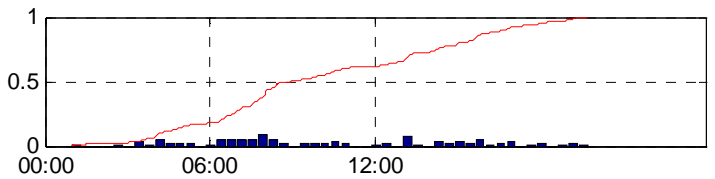

Switch-on instants during a day
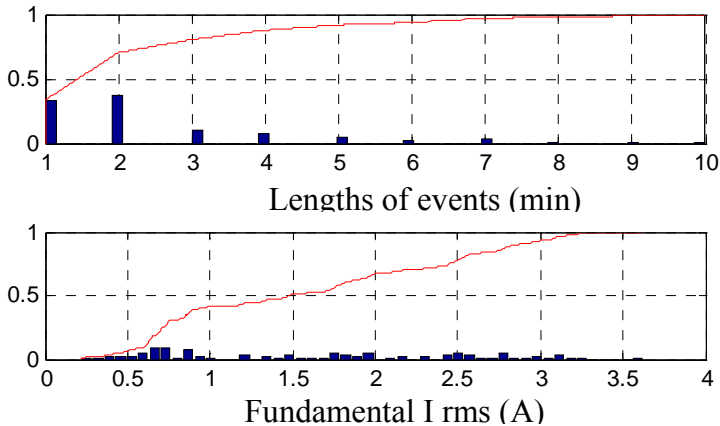

Fundamental I rms (A)

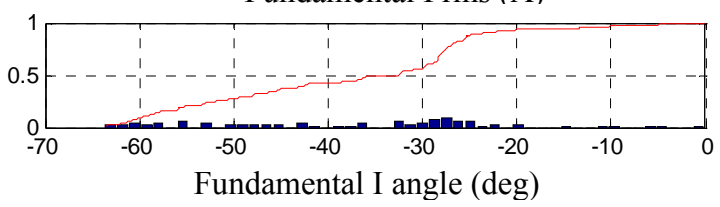

Fig. 4 Empirical cumulative distribution functions of different properties of an "elementary appliance"

Based on these information a random usage pattern having the above properties can be generated, using a uniform random number generator and the inversion method (which now consists of a simple interpolation).

\section{Dirichlet distribution method [4]}

One example use of the Dirichlet distribution is if one wanted to cut strings (each of initial length 1.0) into $\mathrm{K}$ pieces with different lengths, where each piece had, on average, a designated average length, but allowing some variation in the relative sizes of the pieces. The $\alpha / \alpha 0$ values specify the mean lengths of the cut pieces of string resulting from the distribution. The variance around this mean varies inversely with $\alpha 0$.

The application of the Dirichlet distribution for the purpose of distributing the measured currents among the customers is obvious. This method ensures that the sum of the consumer currents equals the measured currents, and that at the same time the mean consumer currents comply with the annual energy consumption of the consumer and usual $\cos \varphi$ and harmonic current ratio values. However, it does not produce realistic consumer current time-functions.

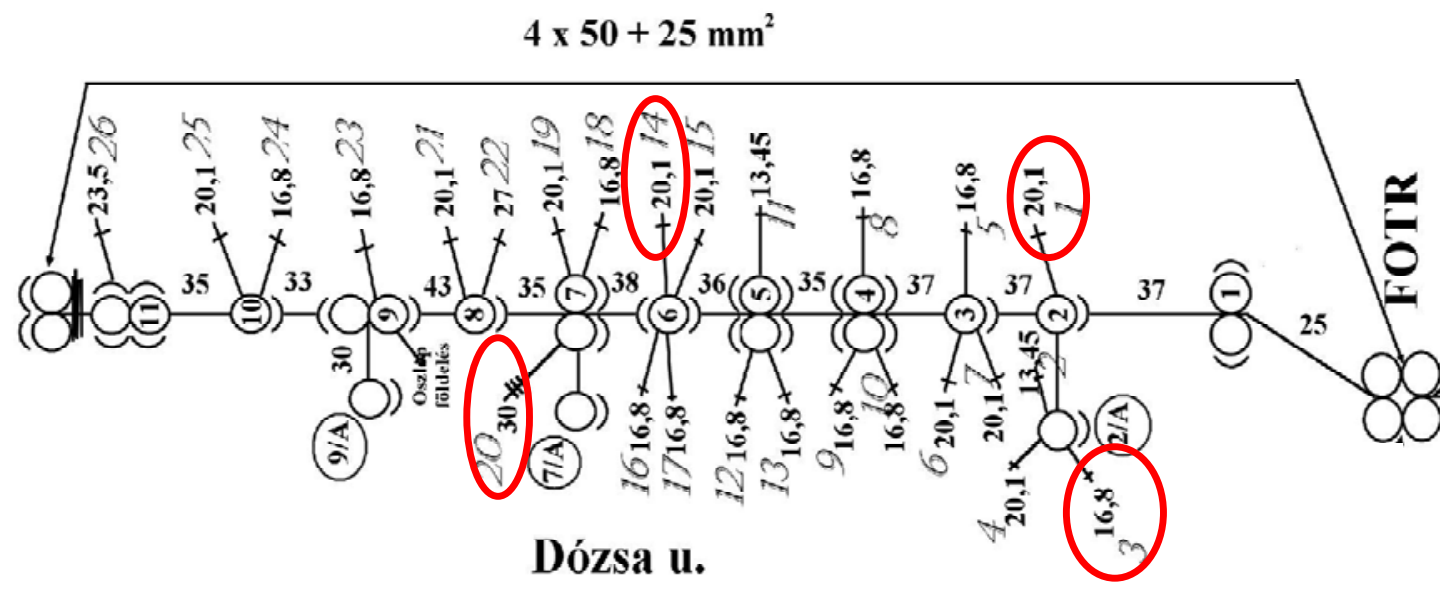

Fig. 5 Simplified scheme of Network 1 (Transformer is denoted FOTR) 


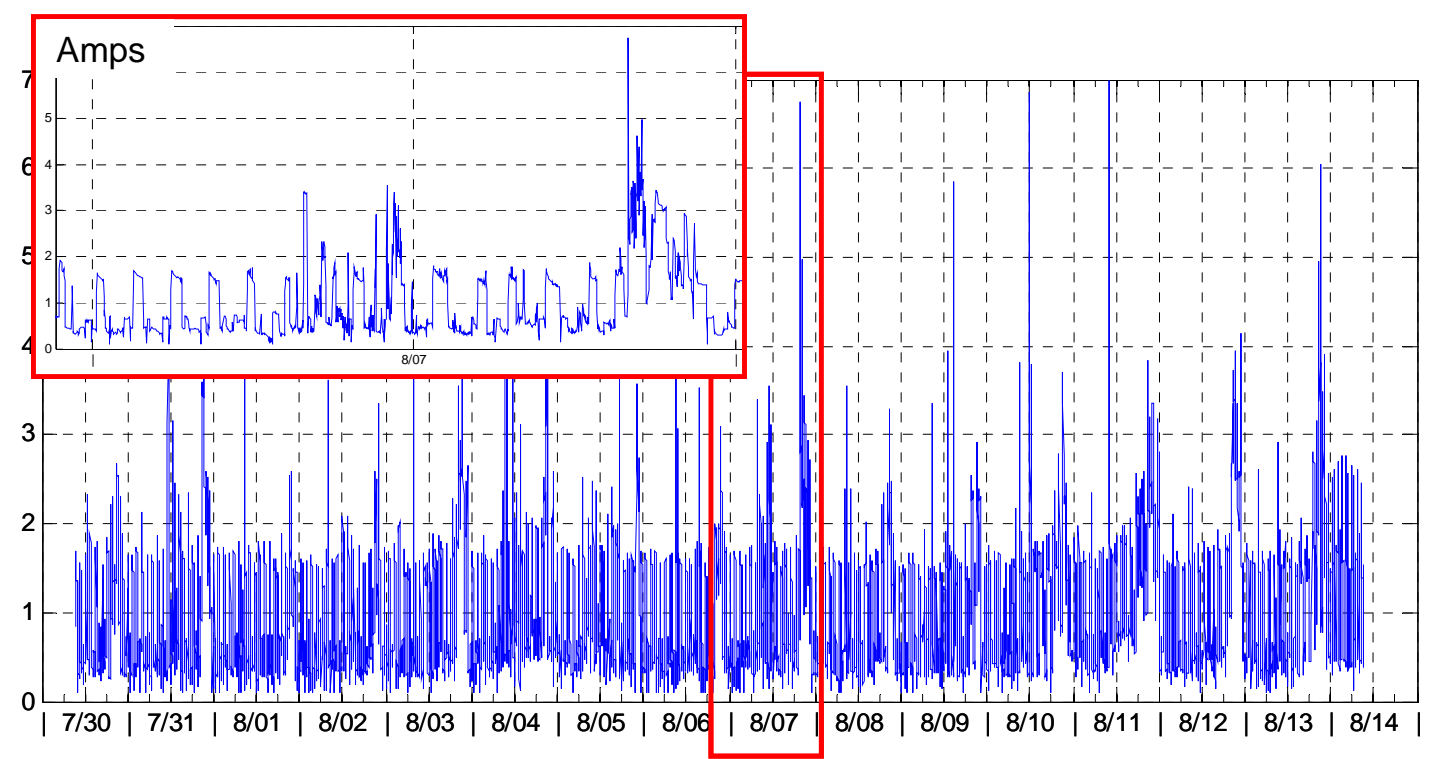

Fig. 6 RMS current measured at one of the households in Network 1 (14 days, 1 day zoomed)

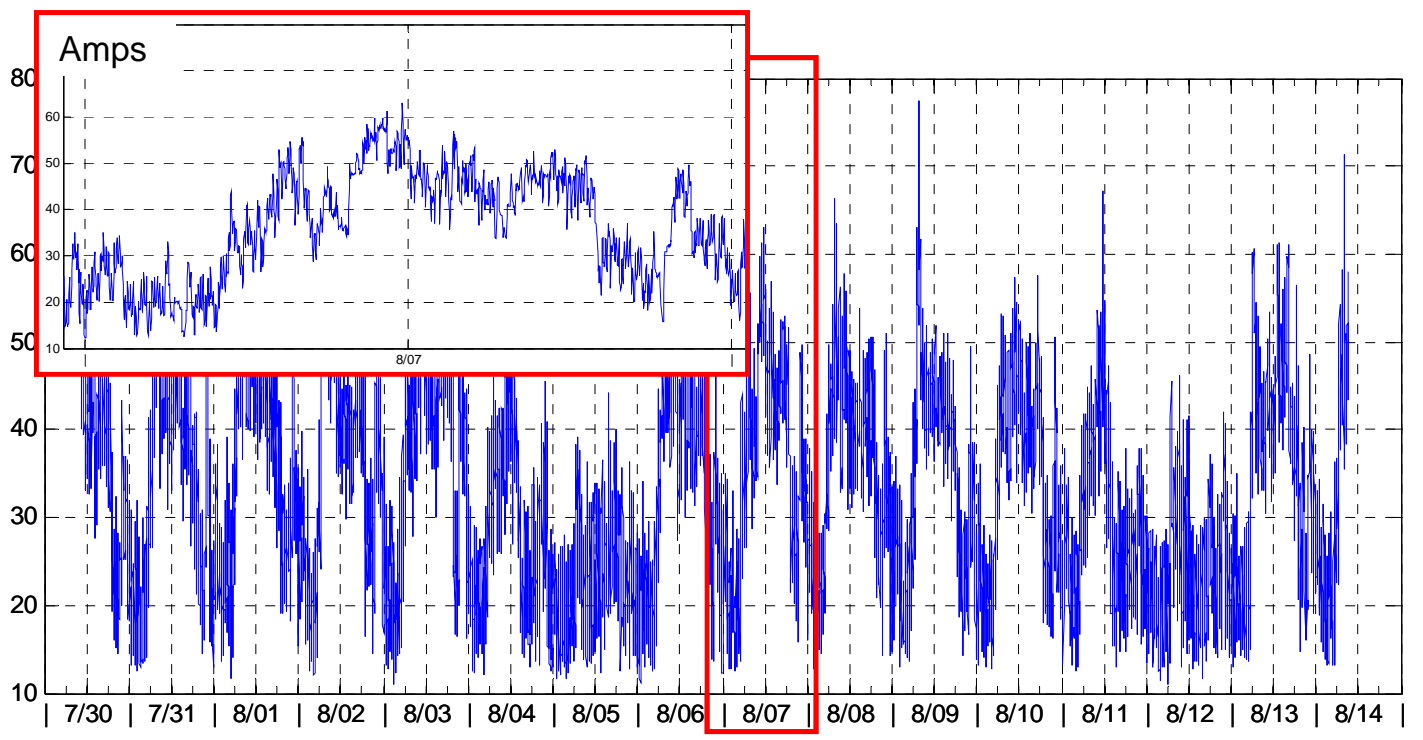

Fig. 7 RMS current measured at the transformer in Network 1 (14 days, 1 day zoomed) 